\title{
Modeling of blood clot removal with aspiration Thrombectomy devices
}

\author{
G. Romero ${ }^{1}$, C. Talayero ${ }^{1,}$, , G. Pearce ${ }^{2}$ and J. Wong ${ }^{3}$ \\ 1 School of Industrial Engineering, Universidad Politécnica de Madrid (UPM), José Gutiérrez Abascal, 2, 28006, Madrid, Spain \\ C/José Gutiérrez Abascal 2, 28006, Madrid, Spain. Phone: +34913363115 \\ 2 Department of Mechanical Engineering, School of Engineering, University of Birmingham, Birmingham, B15 2TT, United Kingdom \\ ${ }^{3}$ Department of Cardiac, Thoracic \& Vascular Surgery, National University Heart Centre Singapore, Singapore.
}

ABSTRACT - Thrombectomy by aspiration is a highly effective method of accomplishing vessel recanalization. This study aims to obtain a mathematical model that allows the prediction of the dynamic response of a thrombus in response to different suction conditions, in order to avoid potential damage or the breakage of the clot during the interventional procedure. Virtual computing models have been created using Bond-Graph data and mass-spring Multi-Degree of Freedom equations. The model allows the use of tensile and torsion loads that could potentially be generated by the suction pressure together with different catheter geometries. The stress generated in the clot depends on its length and on its stiffness. The results obtained with the mathematical model are validated with a Finite Element Method (FEM) model, shows good agreement in terms of stress and elongation values. The results are consistent with previous Bond Graph models which indicated that the forces needed to extract a blood clot from an artery in in-vitro experiments are within the range used experimentally $(\sim 40-90 \mathrm{kPa})$. Qualitative experiments are undertaken with $3 \mathrm{D}$ printed scale prototypes and gelatin. The results are consistent with Computer Fluid Dynamic (CFD) simulations.

\section{ARTICLE HISTORY}

Revised: $18^{\text {th }}$ Nov 2019

Accepted: $18^{\text {th }}$ Nov 2019

\section{KEYWORDS}

Blood clot removal;

modeling;

FEM;

numerical methods.

\section{INTRODUCTION}

Stroke is the third commonest cause of death in the UK with 53,000 deaths having occurred in 2007 alone [1]. Strokes arise in the brain when the blood supply to a given area of the brain becomes disrupted or cut off e.g. with occlusion by a blood clot. The arteries that comprise the Circle of Willis in the human brain are frequently affected by stroke. Treatments for Stroke include anti-coagulants e.g. Alteplase (rTPA), but this can be associated with problems such as risk of bleeding and is contraindicated in some instances such as pregnancy trauma, or postoperatively. Thrombectomy methods [2,3] include Mechanical Thrombectomy Devices (MTDs), aspiration devices or a combination of both [4-11], e.g. the MERCI (Mechanical Embolus Removal in Cerebral Ischemia), the Penumbra device, rheolytic catheters (Angiojet) are used. MTDs, however, may carry potential risks such as breakage of moving parts, risk of potential penetration of the vessel wall, and risks of downstream embolization on account of fragmentation. Suction devices are being developed and different solutions have been patented [12-15]. A thrombo-mechanical extraction named the GPTAD (GP Thrombus Aspiration Device) attempts to overcome some of these potential problems. A schematic view of the GPTAD, is shown in Figure 1.

The device may potentially decrease the risk of downstream embolization because it does not have to make contact with the clot, in order to facilitate blood clot extraction. The GPTAD has no moving parts, thereby this potentially reduces the risk of breakages occurring in the device. The efficacy of the GPTAD in the removal of blood clots has been demonstrated by physical testing previously in other studies [16]. In-vitro studies undertaken by Tennucci et al [17] have also shown that it potentially reduces the risk of clot fragmentation compared to using a straight tube without any helical configuration on the inner surface.

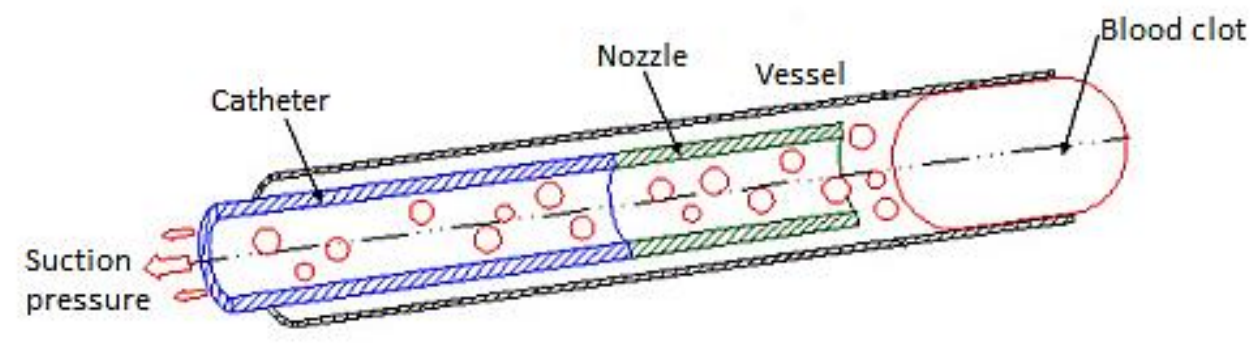

Figure 1. Schematic view of the GPTAD. 
The optimization of the process of clot removal and the geometry of the devices is of paramount importance. For this reason, experiments related to material properties [18], simulation methodologies [19] and nozzle effects [20] have been undertaken. Also, mathematical models have been developed in preliminary studies [21,22] to obtain the appropriate range of different parameters without the need for complex in-vitro or in-vivo experiments. In these studies, the clot removal is modelled with Bond-Graph method, paying attention to the whole system involved in the thrombectomy: pump, catheter, vessel, suction device, blood clot and the existing interfaces. Although these Bond-Graph models facilitated a good approach to some parameters involved in the study, the method is limited especially with regard to the representation of the blood clot, where a high number of equations is needed for obtaining a solution. For that reason, only the conditions regarding the movement of the clot were analyzed (the suction was higher than the resistive load due to the clot-artery wall interaction).

The study we present in this paper aims to predict, with a new mathematical model, the dynamic response of the thrombus with greater accuracy, and studies not only the clot extraction (its movement), but also the behavior of the thrombus. We use virtual models and gelatin prototypes to investigate the minimization of the stress that causes the breakage of the clot and potentially, increases the risk of downstream embolism. Several parameters are investigated such as the size of the thrombus (length and diameter), the contact area between the thrombus and the artery, the pressure of suction, the catheter length and the behavior of the artery. The size and the diameter of the thrombus and artery used in the virtual testing are typically $3 \mathrm{~mm}$ diameter and $30 \mathrm{~mm}$ length (size of Circle of Willis vessels).

\section{METHODS}

The methodology followed is represented in Figure 2. The study begins with a mathematical model that enables to set the blood clot properties and the suction pressure. Representative results are obtained, from which the risk of fragmentation can be obtained (relating to the elongation and the velocity of the clot). In order to validate the mathematical model, a comparison with a FEM model that represents the same blood clot is set up. Finally, tests with gelatin and scaled prototypes are undertaken in order to compare the results qualitatively.

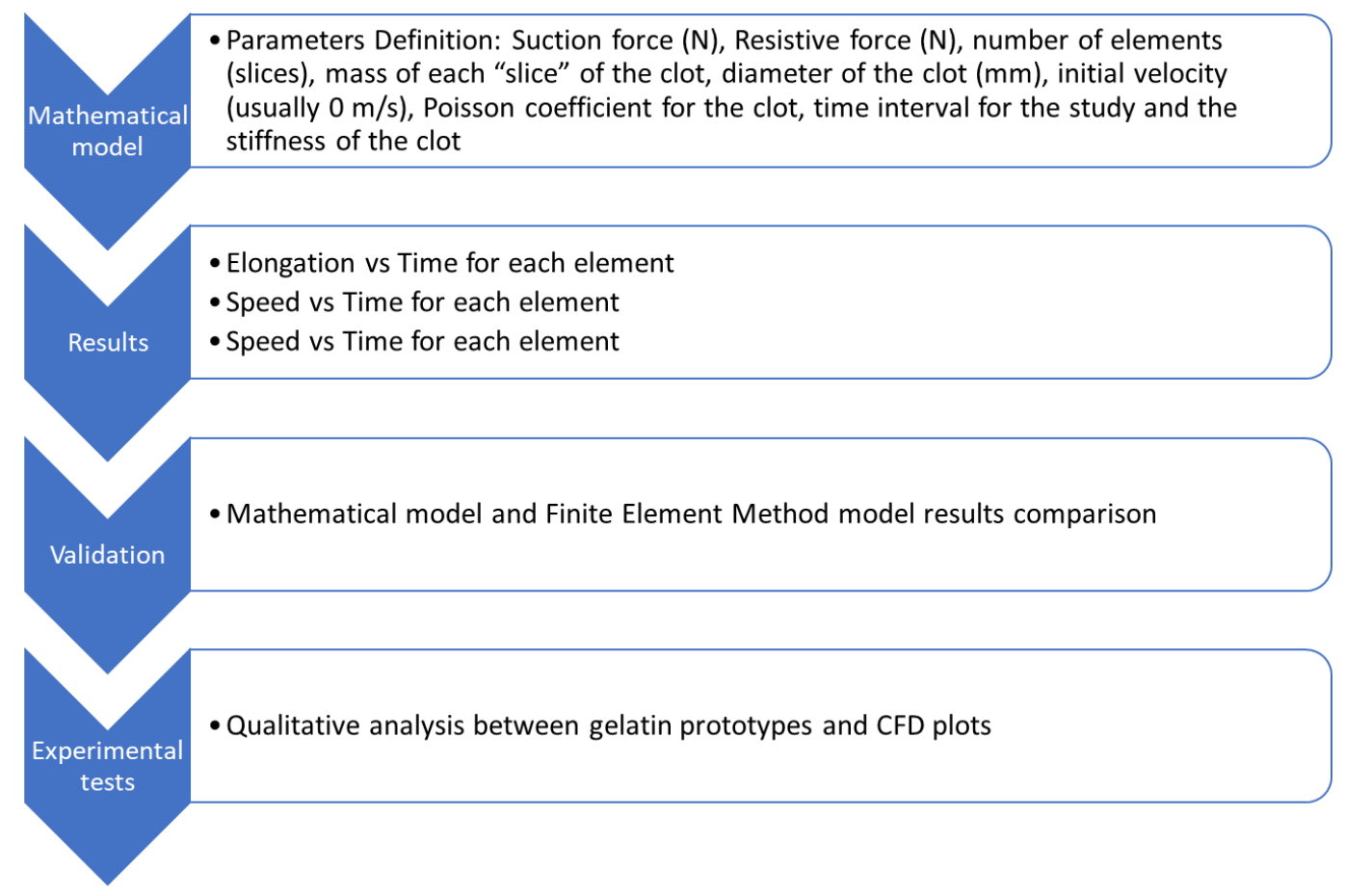

Figure 2. Methodology flowchart.

\section{Mathematical Model}

In the existing studies, we analyze the suction pressure vs time but the clot itself is not analyzed from the point of view of the stresses involved. Using these results (the suction pressure values), we aim to analyze the possible damage to the blood clot, i.e. a model to represent the internal behavior of the clot is considered. We model a clot that is represented as a mass represented as several sections - a spring system that represents "slices" of the clot that can be studied with respect to strain and stress, where the force over the mass is variable in time, $F(t)$ along the direction $x(t)$, and this direction being the only one allowed for the movement of the clot mass. This approach enables us to obtain multiple degrees of freedom system (MDOF). To analyze the strain in the clot zone that is not instantly in contact with the vessel, the proximal end of the thrombus is modelled as a mass-spring system as shown in Figure 3. 


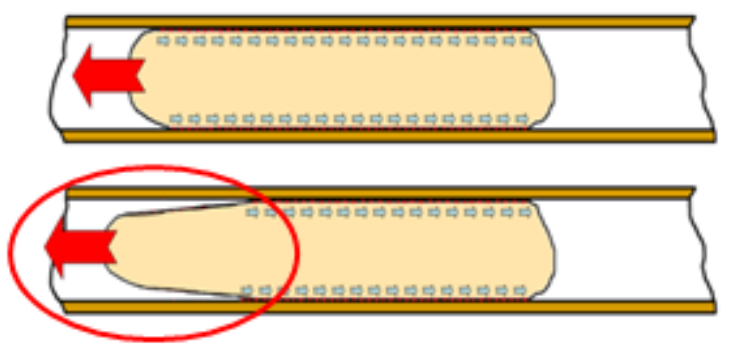

Region to be studied

(a)

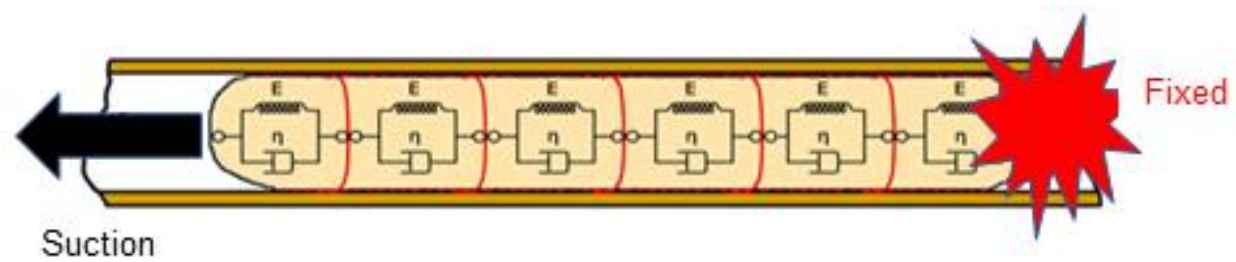

(b)

Figure 3. Clot dynamic model: (a) Clot model and region to be studied and (b) Damped mass-spring system with 6 DOF.

Considering Newton's second law, the result is ' $m \cdot \ddot{x}(t)=k \cdot x(t)+F(t)$, where " $\ddot{x}(t)$ " is the acceleration of the mass at an instant " $t$ " and " $k$ " is the stiffness. According to the model, if the force is applied in a real model, the mass will oscillate with an amplitude that becomes progressively reduced until the movement is halted due to the damping phenomena. This implies an energy loss and is considered as follows, where the updated equilibrium equation corresponds to " $m \cdot \ddot{x}(t)+c \cdot \dot{x}(t)+k \cdot x(t)=F(t)$ ", where " $\dot{x}(t)$ " is the velocity of the mass at the instant " $\mathrm{t}$ ".

For a single degree of freedom, the solution is immediate and, even with a 2 DOF model (that is equivalent to consider the clot as two pieces), a simple differential equation can be used.

The equilibrium equations for the first element are:

$$
-k_{1} x_{1}-c_{1} \dot{x}_{1}+k_{2}\left(x_{2}-x_{1}\right)+c_{2}\left(\dot{x}_{2}-\dot{x}_{1}\right)+f_{1}(x)=m_{1} \ddot{x}_{1}
$$

and rearranging,

$$
m_{1} \ddot{x}_{1}+\left(c_{1}+c_{2}\right) \dot{x}_{1}-c_{2} \dot{x}_{2}+\left(k_{1}+k_{2}\right) x_{1}-k_{2} x_{2}=f_{1}(x)
$$

The governing equation for the second mass is as follows:

$$
m_{2} \ddot{x}_{2}+c_{2} \dot{x}_{2}-c_{2} \dot{x}_{1}+k_{2} x_{2}-k_{2} x_{1}=f_{2}(x)
$$

And in a matrix form:

$$
\left[\begin{array}{cc}
m_{1} & 0 \\
0 & m_{2}
\end{array}\right]\left[\begin{array}{c}
\ddot{x}_{1} \\
\ddot{x}_{2}
\end{array}\right]+\left[\begin{array}{cc}
\left(c_{1}+c_{2}\right) & -c_{2} \\
-c_{2} & c_{2}
\end{array}\right]\left[\begin{array}{c}
\dot{x}_{1} \\
\dot{x}_{2}
\end{array}\right]+\left[\begin{array}{cc}
\left(k_{1}+k_{2}\right) & -k_{2} \\
-k_{2} & k_{2}
\end{array}\right]\left[\begin{array}{l}
x_{1} \\
x_{2}
\end{array}\right]=\left[\begin{array}{l}
f_{1}(x) \\
f_{2}(x)
\end{array}\right]
$$

Notice that with only two elements, the representation of the thrombus is expected to be much stiffer than in reality (similar to a coarse mesh in FEM), so more elements need to be added to the model. The following model is therefore proposed where the matrix formulation is changed into a block representation. The first two elements are shown in Figure 4. To increase the number of elements in the model the scheme of the second element has to replicate up to the last element, that is shown in Figure 5. The size for the studied solid is a typically $3 \mathrm{~mm}$ in diameter and between 10 and 50 $\mathrm{mm}$ in length, corresponding to the dimensions of the Circle of Willis.

The number of elements (slices) needed to be included in the model will be determined by the convergence of the solution, and iterations are needed. Considering a FEM model with linear beam elements, between 5 and 15 elements would give an adequate result. 


\section{First element}

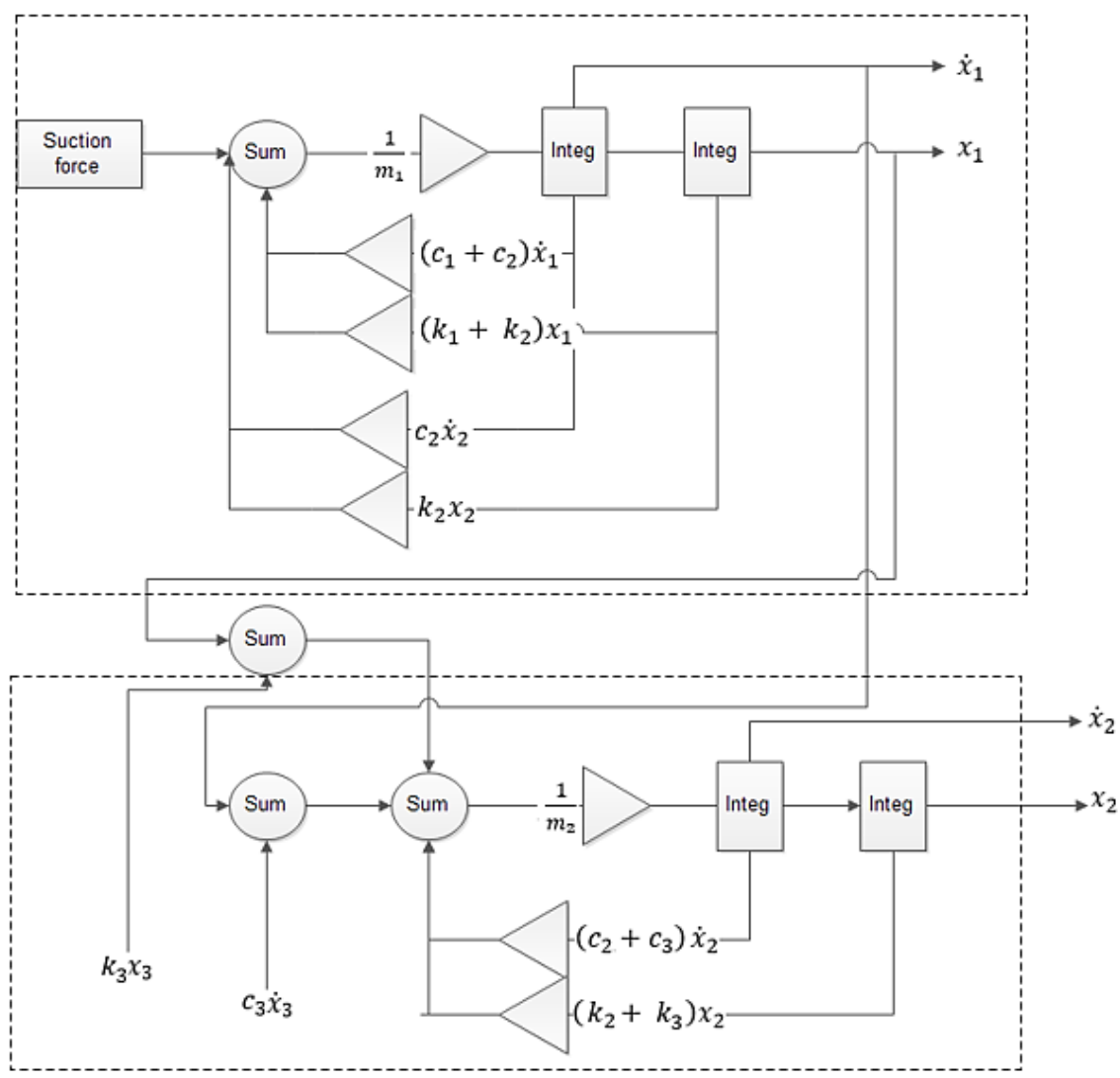

Intermediate element

Figure 4. Model for the $1^{\text {st }}$ and $2^{\text {nd }}$ elements.

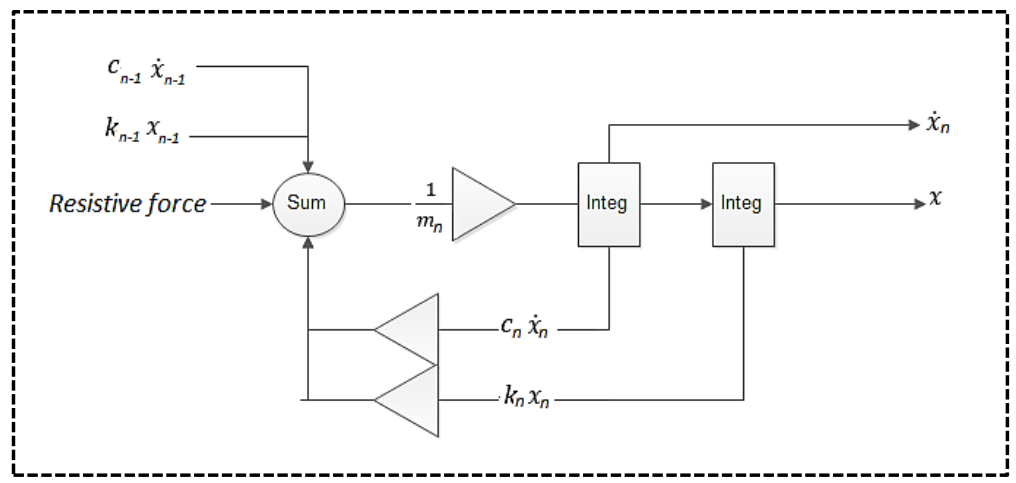

Figure 5. Model for the last (n) element.

\section{Solver}

For solving the model, we use a spreadsheet for convenience and speed, and in order to obtain the status of the system for each instant " $\mathrm{t}$ " and " $\mathrm{t}+1$ ". Furthermore, instead of using the numerical integration, a small enough time lapse is chosen, and the equilibrium equations are solved. To guarantee the convergence and the accuracy of the model, an adequate time interval must be established. In this case, 0.001 seconds is chosen, which means that for a one second study, 1000 status of the system will be solved. The structure of the spreadsheet is as follows: it has an input data tab, an equations tab and the graphic results tab. The fields on the input data tab are: Suction force $(\mathrm{N})$, Resistive force $(\mathrm{N})$, number of elements (slices) that the clot is divided into for the study, mass of each "slice" of the clot, diameter of the clot $(\mathrm{mm})$, initial velocity (usually $0 \mathrm{~m} / \mathrm{s}$ ), Poisson coefficient for the material, time interval for the study and the stiffness of the clot (entered by a force - displacement chart). Since the mass of the system is very small and the speeds are low enough, the damping calculation can be neglected on a first approach. The number of elements is set to six, which gives a good result. The mass of each slice is automatically calculated from the total mass and the number of slices with a total mass of 1 gr chosen. 
The equations are, for the instant for the first element at instant " $t$ ":

$$
\begin{aligned}
& \text { Velocity for instant } \mathrm{t} v[t]_{l}=v[t-1]_{l}+\Delta v[t-1]_{l} \\
& \text { Elongation for instant } \mathrm{t} \quad x[t]_{l}=x[t-1]_{l+} v[t]_{1 . \Delta t} \\
& \text { Force equilibrium } F_{\text {applied }}=F=F_{\text {suction }} \\
& \text { Elongation Force } F_{\text {reaction }}[t]_{l}=-K . \Delta x[t]_{2: 1} \\
& \text { Acceleration for instant } \mathrm{t} a[t]_{I}=\Sigma F / m_{1} \\
& \text { Increment of velocity } \Delta v[t]_{l}=a[t]_{l} . \Delta t \\
& \text { Stress for instant } \mathrm{t} \quad \sigma[t]_{l}=\sigma V M[t]_{l} \\
& \text { Velocity for instant } \mathrm{t}+1 \quad v[t+1]_{l}=v[t]_{l}+\Delta v[t]_{l}
\end{aligned}
$$

and the equations for the " $\mathrm{n}$ " element in the instant " $\mathrm{t}$ " are (in the studied case $1<\mathrm{n} \leq 6$ ):

$$
\begin{aligned}
& \text { Velocity for instant } \mathrm{t} v[t]_{n}=v[t-1]_{n}+\Delta v[t-1]_{n} \\
& \text { Relative velocity } \Delta v[t]_{n: n-1}=v[t]_{n-1}-v[t]_{n}
\end{aligned}
$$

Relative elongation $\quad \Delta x[t]_{n: n-1}=\Delta x[t-1]_{n: n-1}+\Delta v[t]_{n: n-1} . \Delta t$

Force equilibrium $F_{\text {applied }}[t]_{n}=-F_{\text {reaction }}[t]_{n-1}$

Elongation force $F_{\text {reaction }}[t]_{n}=-K . \Delta x[t]_{2: 1}$

$$
\text { Acceleration for instant } \mathrm{t} a[t]_{n}=\Sigma F / m_{n}
$$

$$
\text { Increment of velocity } \Delta v[t]_{n}=a[t]_{n} . \Delta t
$$

Increment of elongation $x[t]_{n}=x[t-1]_{n}+v[t]_{n} . \Delta t$

$$
\text { Stress for instant } \mathrm{t} \quad \sigma[t]_{n}=\sigma_{V M}[t]_{n}
$$

Velocity for instant $\mathrm{t}+1 \quad v[t+1]_{n}=v[t]_{n}+\Delta v[t]_{n}$

For this model, an input variable is the suction force that can vary in a given period of time, depending on the suction pressure and the size of the clot, that can be measured by medical techniques and which was obtained in previous Bond Graph studies. As a reference value, $0.01 \mathrm{~N}$ is considered, and this is reached while suctioning occurs, as shown in Figure 6.

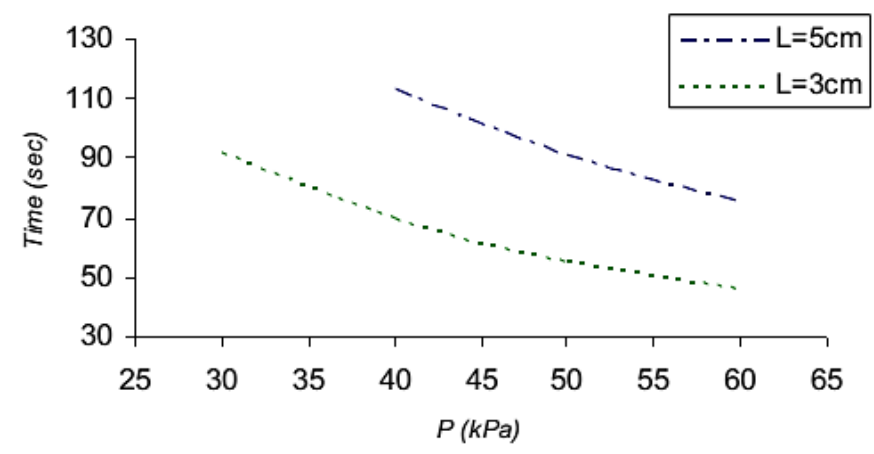

Figure 6. Time for reaching the pressure to initiate the clot movement.

The stiffness of the thrombus is also a variable value depending on the elongation and is typically in the range 0,1 and $0,5 \mathrm{~N} / \mathrm{mm}$ based on experimental testing on porcine thrombus. Two different materials are allowed to consider the superposition of results or a variable stiffness clot. The stiffness of the thrombus is introduced by displacement - force data pairs and a curve is plotted to verify the data. The higher the slope, the stiffer the thrombus. 
The output parameters are the elongation and speed of each element (thrombus slice). Finally, with the force and the elongation having been determined, the stress can be calculated for each slice and for each instant of time. In the first stage, only an axial force is considered, thus the stress will be in tension and in a narrower section (smaller cross-sectional area) that is usually found in the proximal side of the thrombus.

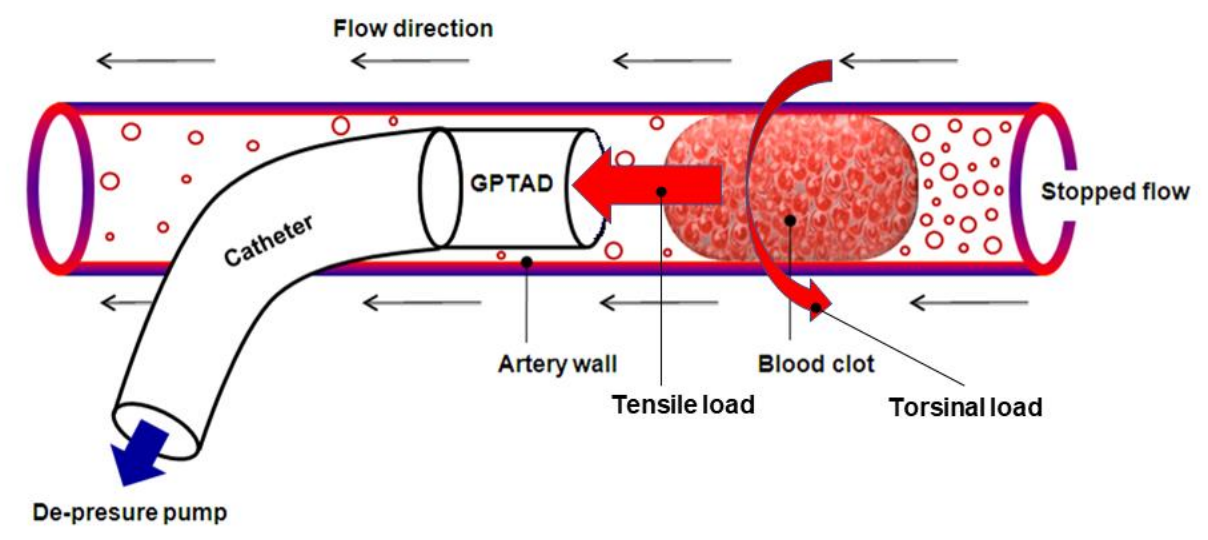

Figure 7. Combined loads applied to the thrombus.

If the pressure were able to generate a torque (Figure 7), a shear load would appear in the bonded section (the thrombus - arterial interface), that is usually far from the suction catheter. The stress on this area is calculated as follows by building the corresponding Mohr's circle (Table 1 and Figure 8).

Table 1. Mohr's circle definition.

\begin{tabular}{|c|c|c|c|}
\hline Description & Symbol & Value / Units & Formula \\
\hline $\begin{array}{l}\text { Normal Stress along } \\
\mathrm{X} \text {-axis }\end{array}$ & $\sigma_{x}$ & $\mathrm{MPa}$ & $\mathrm{F}_{\text {suction }} / \mathrm{A}$ \\
\hline $\begin{array}{l}\text { Normal Stress along } \\
\text { Y-axis }\end{array}$ & $\sigma_{y}$ & $\mathrm{MPa}$ & - \\
\hline $\begin{array}{l}\text { Shear Stress in } \\
\mathrm{XY} \text {-plane }\end{array}$ & $\tau_{x y}$ & $\mathrm{MPa}$ & Torque.r/J \\
\hline Mean Normal Stress & $\sigma_{a v g}$ & $\mathrm{MPa}$ & $\frac{\sigma_{x}+\sigma_{y}}{2}$ \\
\hline Maximum Shear Stress (Tresca) & $\tau_{\max }$ & $\mathrm{MPa}$ & $\sqrt{\left(\frac{\sigma_{x}-\sigma_{y}}{2}\right)^{2}+\tau_{x y}^{2}}$ \\
\hline Maximum Principal Stress (1) & $\sigma_{l}$ & $\mathrm{MPa}$ & $\sigma_{a v g}+\tau_{\max }$ \\
\hline Minimum Principal Stress (2) & $\sigma_{2}$ & $\mathrm{MPa}$ & $\sigma_{a v g}-\tau_{\max }$ \\
\hline Von-Mises Stress & $\sigma_{V M}$ & $\mathrm{MPa}$ & $\sqrt{\left(\frac{\left(\sigma_{1}-\sigma_{2}\right)^{2}+{\sigma_{2}}^{2}+\left(-\sigma_{1}{ }^{2}\right)}{2}\right)^{2}}$ \\
\hline
\end{tabular}




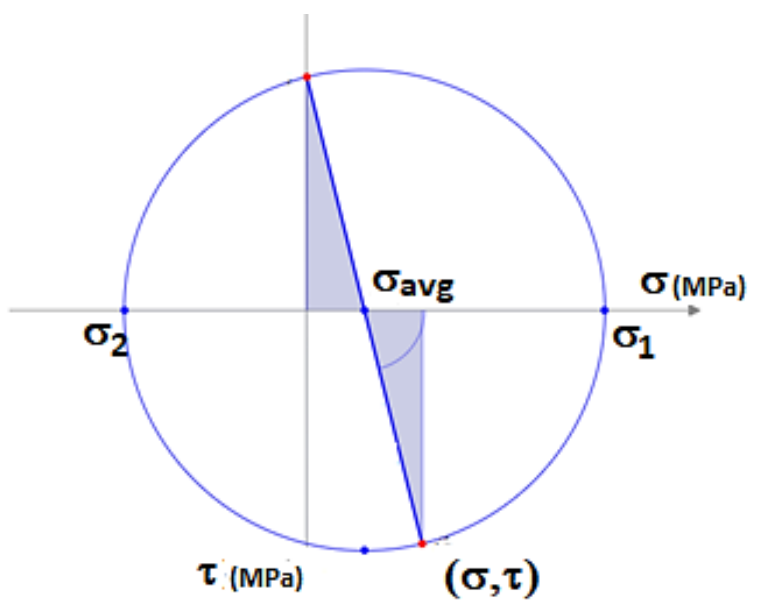

Figure 8. Mohr's circle for Normal and shear stress.

A combination of values of axial load and torsional load is considered, assuming that it is possible to create such a torque with a catheter with a helical geometry. For this study, and in order to analyze the beginning of the movement of the thrombus, a resistive force slightly higher than the suction force is chosen. This corresponds to a state when the maximum suction pressure is reached (force) since in the next instant of time, the resistive force will be released and the stresses will drop significantly until the next event occurs, namely, the collision with the catheter. The value is consistent with those obtained in in vitro testing [17].

\section{Validation}

In order to validate the results obtained by the mathematical model, a FEM study has been undertaken keeping the same parameters: $3 \mathrm{~mm}$ of diameter, 10 and $30 \mathrm{~mm}$ length. As described in the section "Solver", a shear stress will appear if the pressure were able to create a torque high enough to torsion the thrombus. So, in order to analyze this with greater accuracy, five different helical catheters have been designed. To verify that the resistive force is as according to the material chosen properties and to the geometry, the contact area has been modelled as a pressure fitting according to the following expression:

$$
p=\frac{\delta}{\frac{d}{E_{0}}\left(\frac{d_{0}^{2}+d^{2}}{d_{0}^{2}-d^{2}}+v_{0}\right)+\frac{d}{E_{i}}\left(\frac{d^{2}+d_{i}^{2}}{d^{2}-d_{i}^{2}}+v_{i}\right)}
$$

where $\delta$ is the radial interference, $\mathrm{d}$ is the nominal diameter, $\mathrm{d}_{\mathrm{i}}$ is the inner diameter of the male component (the thrombus in this case, and it is equal to zero), $d_{o}$ is the outer diameter of the hub (the vessel in this case), $E_{o}$ and $E_{i}$ are the Young modulus for the outer and inner components (vessel and thrombus) and $v_{\mathrm{o}}$ and $v_{\mathrm{i}}$ are the Poisson coefficients for the vessel and the thrombus respectively. The following values are chosen:

Table 2. Parameters definition.

\begin{tabular}{ll}
\hline Parameter & Value \\
\hline$\delta$ & $0.4 \mathrm{~mm}$ \\
$\mathrm{E}_{0}$ & $0.11 \mathrm{MPa}$ \\
$\mathrm{E}_{1}$ & $0.06 \mathrm{kPa}^{*}$ \\
$\mathrm{~d}$ & $3 \mathrm{~mm}$ \\
$\mathrm{~d}_{0}$ & $5 \mathrm{~mm}$ \\
$\mathrm{~d}_{1}$ & $0 \mathrm{~mm}$ \\
$\mathrm{v}_{\mathrm{o}}$ & 0.45 \\
$v_{\mathrm{i}}$ & 0.49 \\
\hline
\end{tabular}

* For the estimation of the radial pressure, a value as isotropic has been considered for the thrombus

The applied loads are the same as in the mathematical model. Since the clot is a mass that has usually formed in another location, usually in a vascular artery, or in the heart, and has become dislodged becoming trapped in smaller diameter arteries, such as the cerebral artery (considered in this work), we will consider the clot as a hyperelastic solid instead of as a Non-Newtonian fluid (as blood is usually modeled). Hyperelastic material models can be used to model materials such as rubber, where the solutions involve big strains. We therefore assume an elastic material, which is nonlinear, isotropic and incompressible. This incompressibility causes numerical difficulties in the element formulation and it is corrected with the introduction of a compensation based factor on a compressibility in the strain energy density 
function, that is used in the assembly of the additional DOF into the stiffness matrix. This penalty function changes the original function from incompressible to nearly incompressible. In particular, the Mooney Rivlin model strain energy density function is:

$$
\begin{gathered}
W_{1}=A(I-3)+B(I I-3)+X\left(\frac{1}{I I I^{2}}-1\right)+(I I I-1)^{2} \\
W_{2}=C(I-3)(I I-3)+D(I-3)^{2}+E(I I-3)^{2}+F(I-3)^{3} \\
W=W_{1}+W_{2}
\end{gathered}
$$

where I, II, and III are invariants of the right Cauchy-Green strain tensor and can be expressed in terms of principal stretch ratios. A, B, C, D, E, and F are Mooney material constants in stress dimensions. For the model studied, a two constants formulation has been chosen, so A and B are considered (first and second constants).

For the mesh of the thrombus and the vessel, tetrahedral elements (TETRA10) have been used with a size that adequately represents the geometry and that reproduces correctly the bending effects, especially in the artery. The chosen high order elements also provide better stability in the hyperelastic model than lower order elements (TETRA 4). Additionally, a coarse mesh will generate a stiffer system that will not represent adequately the aspiration thrombectomy intervention. The geometry and the mesh are shown in Figure 9. The chosen properties for the materials are shown in the Table 3.

Table 3. Material properties.

\begin{tabular}{ll}
\hline Vessel (artery) & Thrombus \\
\hline Isotropic linear & Hyperelastic Mooney Rivlin model \\
Young's modulus E $=0.11 \mathrm{MPa}$ & Material first constant $(\mathrm{A})=4$ \\
Poisson $=0.45$ & Material second constant $(\mathrm{B})=2.5$ \\
Yield limit $=3 \mathrm{MPa}$ & Poisson $=0.49$ \\
\hline
\end{tabular}

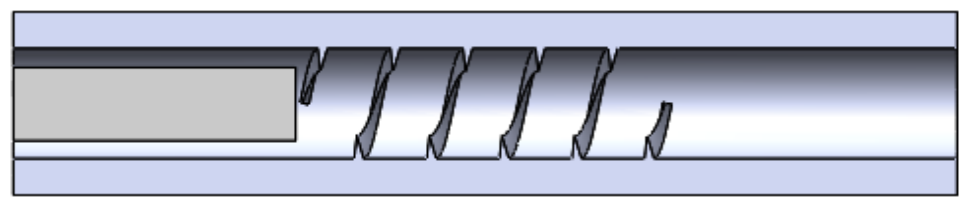

(a)

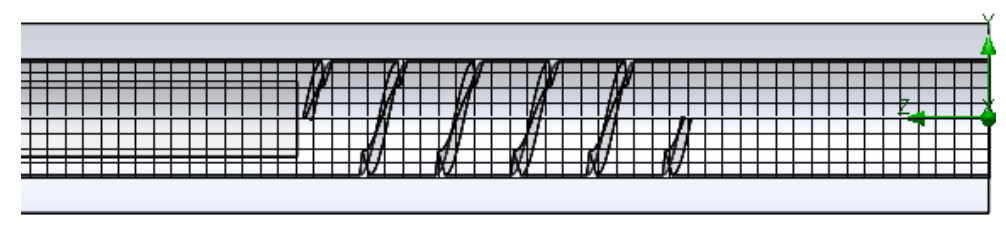

(b)

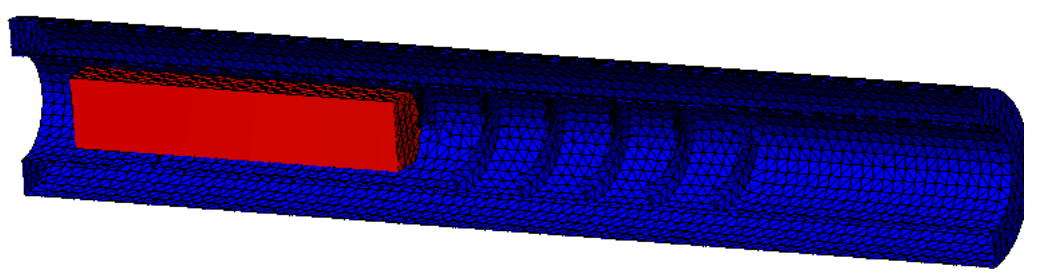

(c)

Figure 9. Helix + Clot: (a) 3D Model, (b) Mesh for CFD and (c) Mesh for nonlinear FEM.

The dynamic analysis was simulated with SolidWorks Nonlinear Dynamics and with SolidWorks Flow Simulation since these allow us to model the thrombectomy event during a period of time. The boundary conditions for the study are:

- Fixed artery on the endings, far enough from the area being studied (artery ending distance to the thrombus is three times the length of the thrombus), to reproduce the strain properly.

- Zero initial velocity on the distal thrombus surface. 
- An interference fit between the vessel and the clot of $0.04 \mathrm{~mm}$ with a static friction coefficient $(\mu)$ of 0.4 along $6 \mathrm{~mm}$, has been modelled for reproducing the resistive force. For both $10 \mathrm{~mm}$ and $30 \mathrm{~mm}$ lengths of thrombus, the same interference has been modelled. This has been estimated from Equation. (23).

In addition, an automatic time lapse has been chosen, initially using $0.01 \mathrm{sec}$, and varying from $1 \mathrm{e}-6$ to 0.01 seconds; allowing up to 5 iterations on each time step. These dynamic parameters allow us to simulate the thrombectomy event as it would appear under real conditions.

\section{Experimental Investigations: Blood Clot Extraction}

To verify the behavior of the clot and the dependence of the suction force on the catheter geometry, some experiments have been undertaken where gelatin was used to represent the blood clot. Gelatin was used to represent the blood clot, because both gelatin and blood clot are composed of liquid and solid particles that produce a deformable solid; the clot being formed by platelet aggregation, and gelatin by water and gelatin powder. It was possible to choose the density of the gelatin by modifying the quantity of gelatin powder and in the experiments the density of the gelatin was about $20 \%$. That quantity of gelatin powder was put in hot water, mixed, and placed it in a refrigerator until the gelatin was solid about 45 minutes; after that, the gelatin was inserted in a transparent plastic tube of $25 \mathrm{ml}$ with graduations, which was closed on one side. The gelatin filled the entire diameter of the tube to reproduce a $100 \%$ blood clot occlusion of the artery. A transparent tube was connected to a syringe and the whole assembly was filled with water. The syringe was pulled back to extract the blood clot. In order to observe the movement of the clot, lines of ink were injected into the gelatin as shown in Figure 10, mixing the ink with alcohol to make it more stable. In addition, to create a difference of pressure and so a suction force, we used a $25 \mathrm{ml}$ plastic syringe of $25 \mathrm{ml}$ graduation to suck the gelatin up by pulling the piston. Finally, all pieces were assembled together and placed into water at 37 degrees centigrade. The experiments were undertaken in water to avoid air inflow.

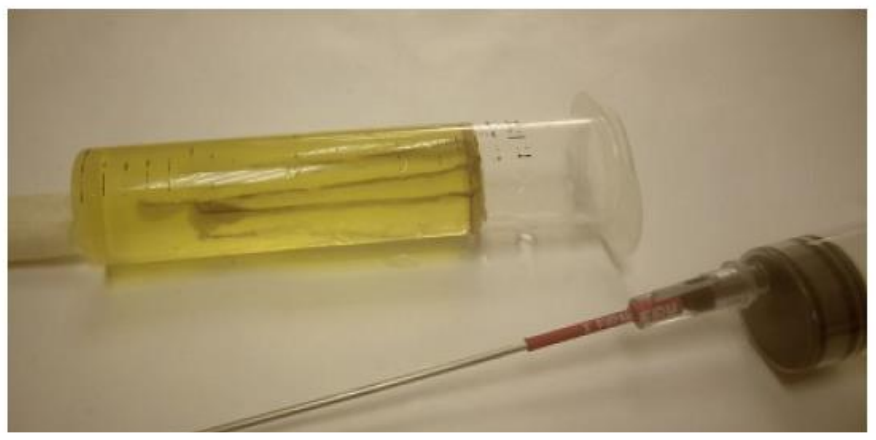

Figure 10. Experiment set up, gelatin with lines of ink.

3D printed models have been manufactured using a BQ Witbox model which uses the Fused Filament Fabrication (FFF) method to print by adding melted polymer filament layer by layer from a print base; the filament, made of polylactic acid (PLA) - a thermoplastic polyster - had a thickness of $1.75 \mathrm{~mm}$. In addition, the software Cura v10 has been used to create the 'gcode' printing file - format - from the 3D design object; to make the object solid. These are shown the following figure:

\section{RESULT AND DISSCUSSION}

The results for the mathematical model are shown in Figures 11 and 12. Figure 11 shows the elongation vs time for each element (slice) of the clot, having considered the case for studying six elements (slices). The movement follows a dual slope law, where the first part with a steeper gradient, is affected in particular by all the slices at the start of movement (wave forms), and the second, almost straight, is led by the elastic behavior of the clot. Figure 12 shows the stress (tensile) vs time for each element (slice) of the clot. It is noted that the variation in time is due to the "spring effect" of each slice; the movement is as follows: the first element is strained until the elastic force leads the second element to begin movement. Since the second element has stored energy, at the beginning of the movement it moves faster than the first element. This implies a reduction of the strain and, as a consequence, a stress reduction. This occurs each time an element begins to move. It is notable that the last element, even without having begun the movement, is stressed and, for the applied load, is in equilibrium. In this case, a little higher load will initiate movement. The total elongation of the clot of $10 \mathrm{~mm}$ is 2,8 $\mathrm{mm}$ reached 5 seconds after the pressure reaches $30 \mathrm{kPa}$. 


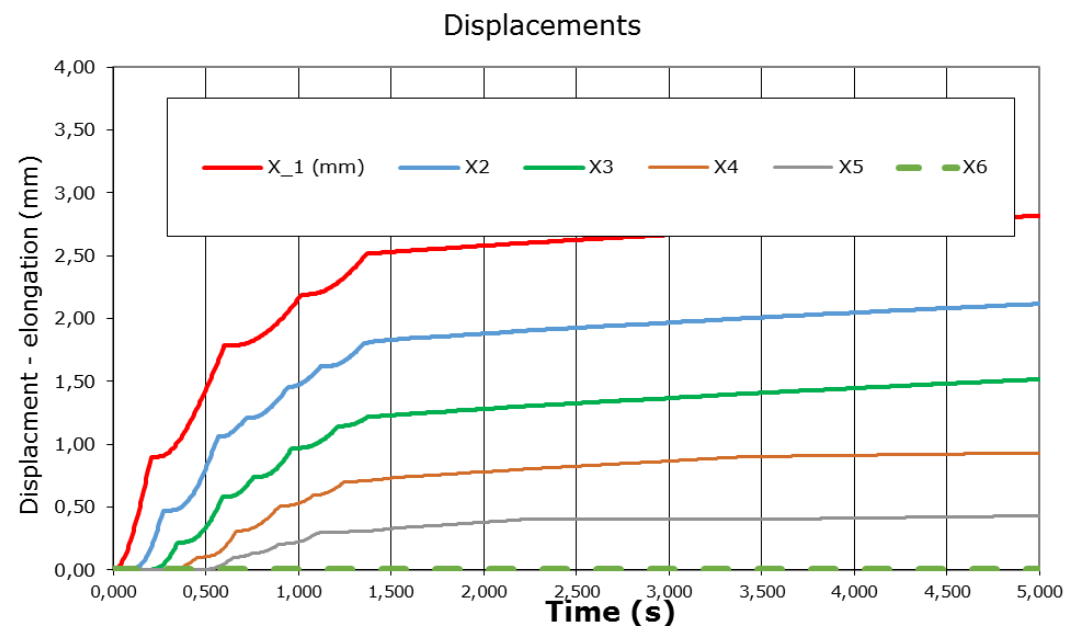

Figure 11. Displacement of each element (6 elements model).

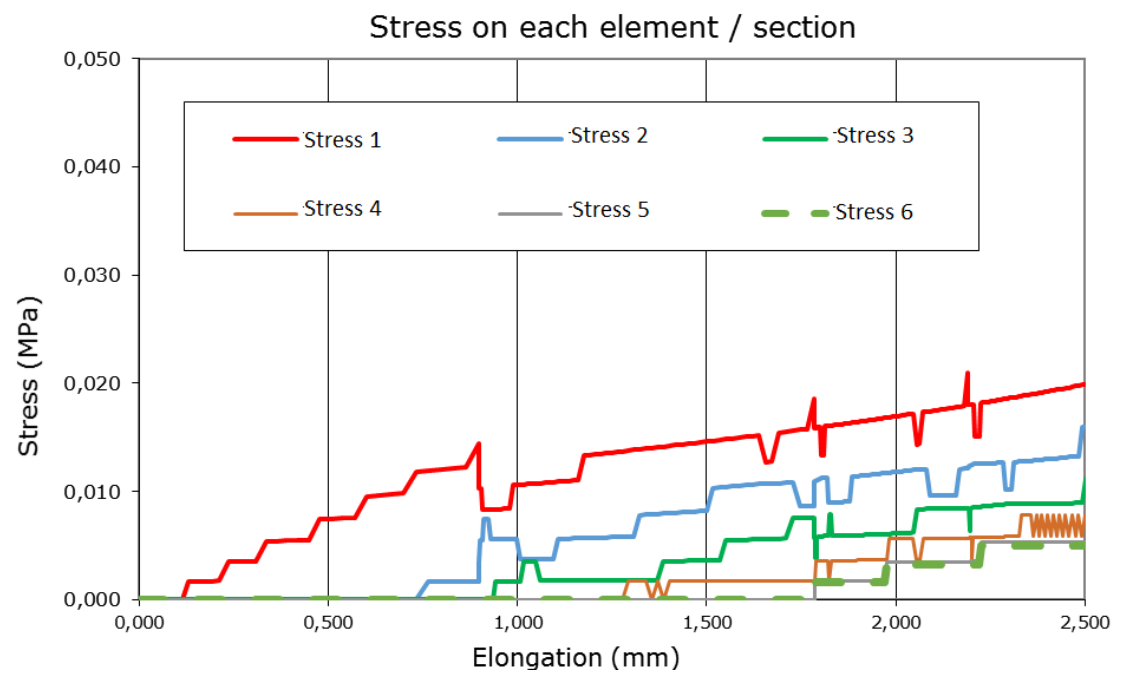

Figure 12. Stress on each element (6 elements model).

Five different suction scenarios have been evaluated with the developed tool (Fig13), combining traction and torsion in different proportions. As expected, in both cases, for both lengths, for a prevailing tensile load (low rotational component) the maximum stress is obtained in the same zone, that is, the load application face. Since the $10 \mathrm{~mm}$ thrombus is stiffer than the $30 \mathrm{~mm}$ one, the transverse section reduction is less significant.

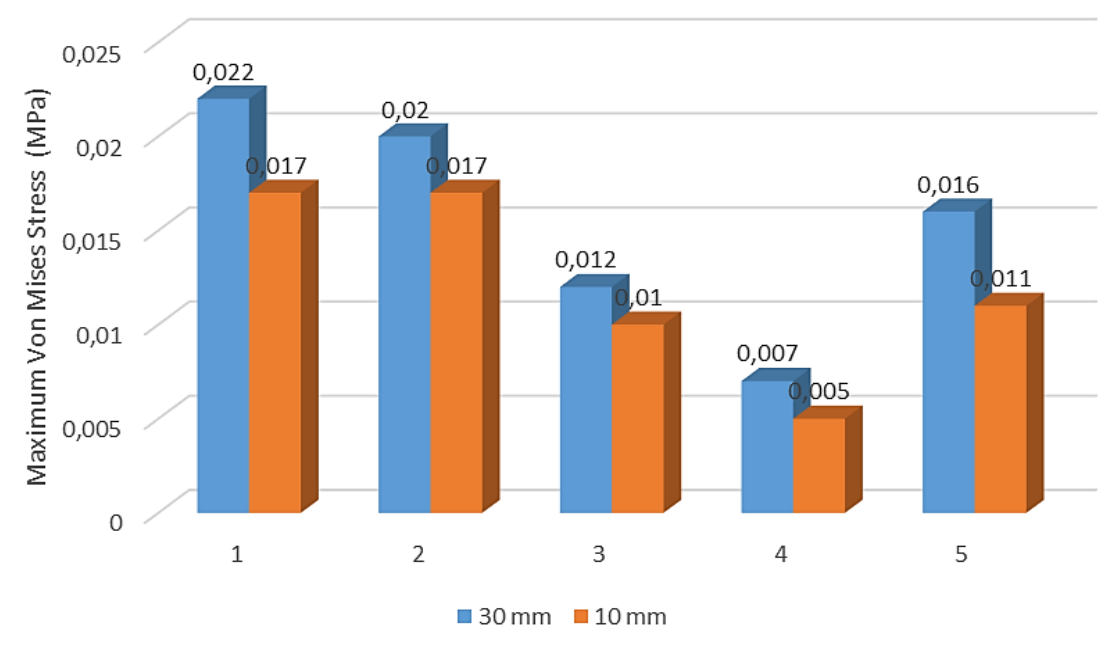

Figure 13. Stress maximum values for 30-10 mm clot length for five helix geometries. 
The validation gives a good correlation with the FEM models, as shown in Figure 14, where the maximum stress of the $10 \mathrm{~mm}$ clot is $0,019 \mathrm{MPa}(0,017 \mathrm{MPa}$ in the FEM model) with an elongation of 1,68 mm, and the maximum stress for the $30 \mathrm{~mm}$ clot is $0,027(0,022$ in the FEM model) with an elongation of 3,22 mm.

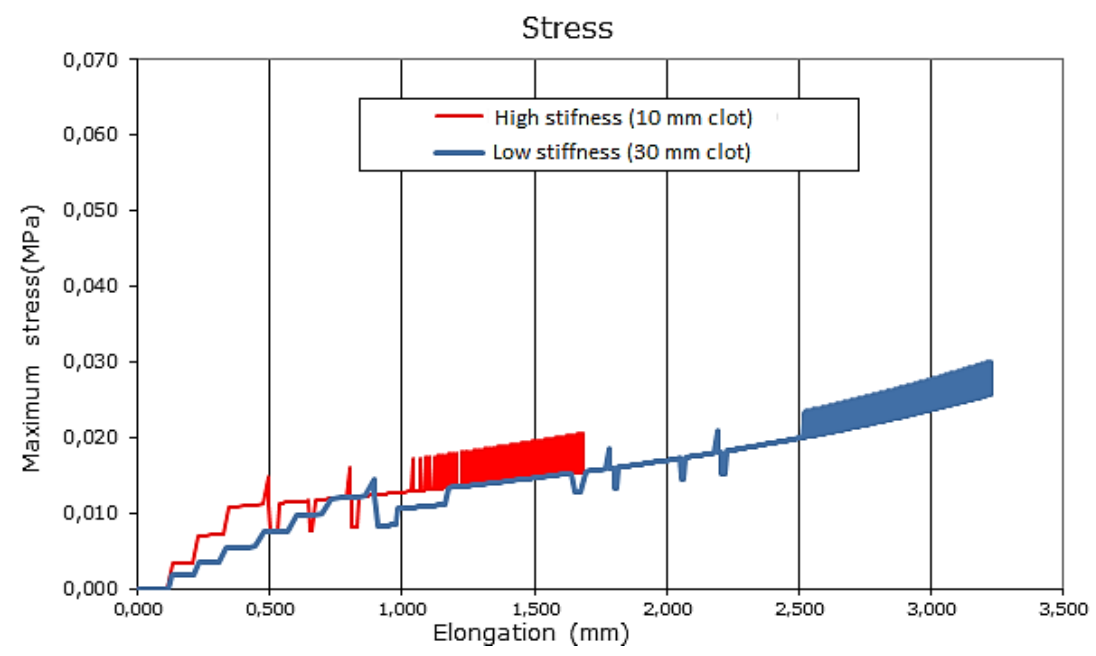

Figure 14. Stress - elongation graph for $10 \mathrm{~mm}$ and $30 \mathrm{~mm}$ clot length.

The results obtained using the CFD model are shown in the figures below where the pressure profile is shown. As can be seen, a rotational component appears due to the helical geometry that will create a shear load in the clot - arterial interface.

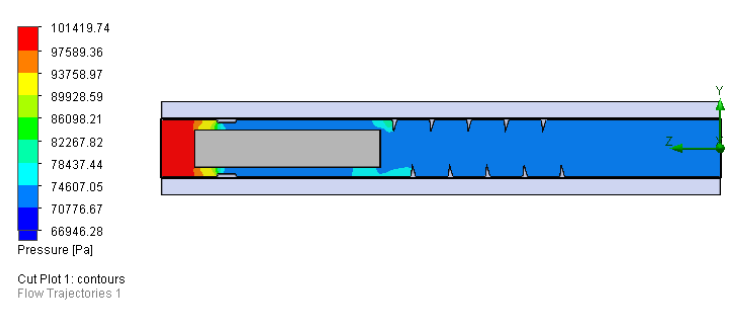

(a)

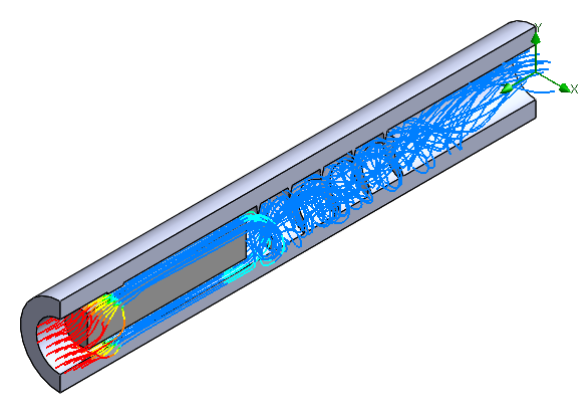

(b)

Figure 15. Pressure field: (a) Section cut plot, (b) Isometric view- Trajectories plot.

The results of the laboratory based experiments are shown in Figure 16. When the syringe was pulled to create the suction force, the gelatin moved linearly in the device as expected. Once the gelatin started moving, its velocity seemed to be constant and in a longitudinal direction. However, it is observed that the gelatin twisted (see ink lines in figure) due to the torsional effect associated with the existing helix, which is reflected in the FEM simulation modeling results.

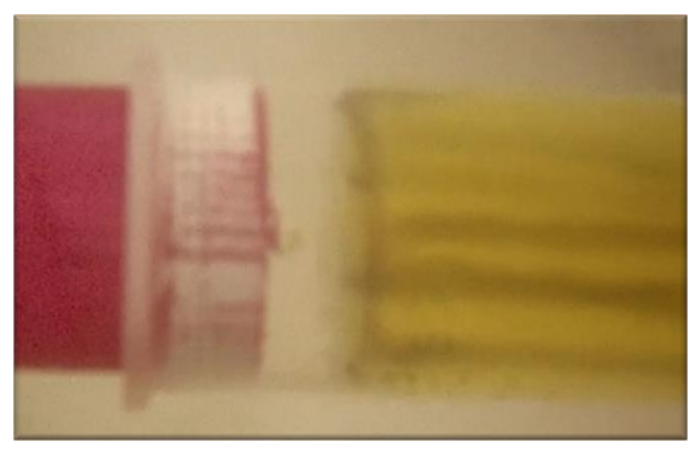

(a)

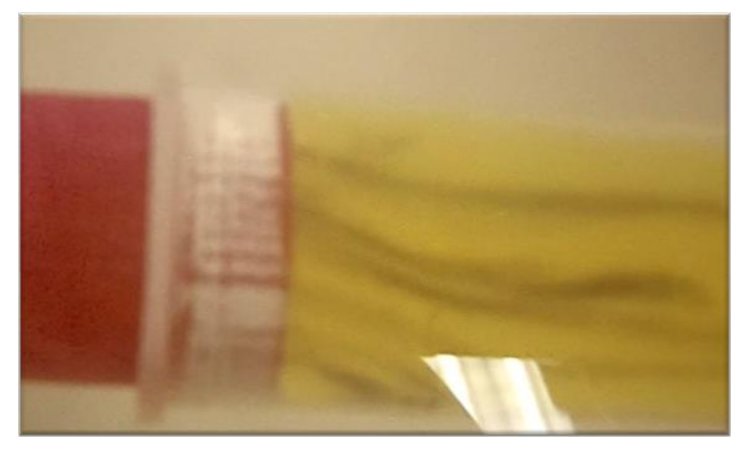

(b)

Figure 16. Gelatin absorption: (a) Before suction and, (b) Rotational clot deformation. 
The results obtained are consistent with previous Bond Graph models that indicated that the forces needed to extract a blood clot from an artery in in-vitro experiments are within the range $440-90 \mathrm{kPa}[21,22]$. This indicates that the GPTAD may therefore have potential use in the removal of blood clots in human arteries because it will not potentially damage the arteries due to the suction operating pressure. The spring-mass system is useful to study the behavior of blood clots in arteries and potentially provides a realistic approach to clot behavior (and any potential damage or clot fragmentation) during the clot suction stage (elongation and stress). The model developed also has the potential to provide realistic results concerning the influence of the stiffness of the clot in relation to any potential damage.

Based on these pressure values and resistive loads, complex geometries can be designed and virtually tested with the combination of Computer Fluid Dynamic and Nonlinear Dynamic FEM models. The 3D based models provide us with a detailed analysis of suction force (based on a pressure field). The results of mathematical models and FEM models are consistent (stresses and elongation). It is significant that the mean solving time for the computer models are around 120 min in a Core 17 processor while the spreadsheet is updated in less than a second for new values.

The analysis of the results allows to draw some conclusions about the behavior of the thrombus. Firstly, the longer the thrombus, the higher the stress, that arises on account of the lower stiffness. When tensile loads are predominant (low helix influence), very high elongation is produced and as a consequence, the section reduction causes higher stress. On the other hand, lower stiffness results, when a prevailing torque (high helix influence), provokes higher angular strain and, therefore, higher stress. This can be appreciated in the bar diagram shown in Figure 13.

The most favorable load combination for both $10 \mathrm{~mm}$ and $30 \mathrm{~mm}$ thrombus (the lowest stress value) are obtained for the Helix $\mathrm{n}^{\circ} 4$, where an adequate combination of axial suction and rotation is achieved. When combining tensile and torque loads (corkscrew / twister effect), torsion occurs, and clot movement is initiated by overcoming the interface resistive loads, and the axial suction load manages the longitudinal movement. A pure torque application will only give a torsion that will not lead to the clot extraction, while a pure traction will suction the thrombus by breaking it into pieces close to the catheter, where the maximum stress values are observed.

The gelatin experiments showed the rotational effect caused by the helix and the necessity of creating a good seal between the GPTAD device and artery wall to avoid inflow and increase the suction effectiveness over the blood clot.

\section{CONCLUSIONS AND FUTURE WORK}

The studies and experiments lead us to conclude that:

- The stress resultant from the required suction pressure to remove a blood clot in an artery as derived from the massspring model are consistent with those produced by experimental investigations and previous studies.

- The stiffer the blood clot, the better its extraction, with respect to clot breakage.

- A geometry that creates a rotational effect combined with axial suction could be better for suction than straight cylindrical ducts. This effect can be created by a helix section positioned within the catheter.

- 3D based models are appropriate for solving these problems where movement of both fluids and solids is involved in combination.

- Mass-spring systems are suitable for solving elastic solid transient problems. A small time-lapse must be set however, and the number of elements to obtain the convergence of the model will depend on the accelerations involved in the system.

- For the studied system, due to the low accelerations values and to the very small mass, the damping effect can be neglected.

- The gelatin experiments validated the torsional effect of the GPTAD device.

- The GPTAD is worthy of further investigation as a potential blood clot removal device.

- The beginning of the movement of the clot in an aspiration thrombectomy has been studied, obtaining the suction pressure and clot behavior for cylindrical and helical distal end geometries. It is evident that the influence of the distal end is an important factor in the behavior of the clot. In future studies, we propose to undertake studies to optimize the suction process and the influence of the distal end of the catheter

\section{ACKNOWLEDGMENT}

The authors would like to thank Adéle Gillier, $1^{\text {st }}$ year Engineering-student at École des Ponts (under an internship carried out in the research group), for producing the printed models used in the research.

\section{REFERENCES}

[1] Scarborough P et al. Stroke Statistics. British Heart Foundation and Stroke Association. . Department of Public Health, University of Oxford. 2009:12-13.

[2] Spiotta AM, Chaudry MI, Hui FK,et al. Evolution of thrombectomy approaches and devices for acute stroke: A technical review. Journal of Neurointerventional Surgery. 2015;7(1):2-7.

[3] Hesse AC, Behme D, Kemmling A, Zapf A, Hokamp NG, Frischmuth I et al. Comparing different thrombectomy techniques in five large-volume centers: A "real world" observational study. Journal of NeuroInterventional Surgery 2018; 10(6):525-529.

[5] Son S et al. Comparison of Solitaire thrombectomy and Penumbra suction thrombectomy in patients with acute ischemic stroke caused by basilar artery occlusion. Journal of Neurointerventional Surgery. 2014; 8(1):13-18. 
[6] Mokin M, Ionita CN, Nagesh SVS, Rudin S, Levy EI, Siddiqui AH. Primary stentriever versus combined stentriever plus aspiration thrombectomy approaches: In vitro stroke model comparison. Journal of Neurointerventional Surgery 2015; 7(6):453457.

[7] Mokin M, Nagesh SVS, Ionita CN, Mocco J, Siddiqui AH. Stent retriever thrombectomy with the Cover accessory device versus proximal protection with a balloon guide catheter: In vitro stroke model comparison. Journal of Neurointerventional Surgery. 2016; 8(4):413-417.

[8] Davis S, Lees K, Donnan G. Treating the acute stroke patient as an emergency: current practices and future opportunities. International Journal of Clinical Practicews. 2006; 60(4):399-407.

[9] Gobin YP, et al. MERCI 1: A phase 1 study of mechanical embolus removal in cerebral ischemia. Stroke. 2004; 35(12):28482854.

[10] Bose A et al. The penumbra system: A mechanical device for the treatment of acute stroke due to thromboembolism. American Journal of Neuroradiology 2008; 29(7):1409-1413.

[11] Fanous AA, Siddiqui AH. Mechanical thrombectomy: Stent retrievers vs. aspiration catheters. Cor et Vasa. 2016;58(2):193-203.

[12] Lapergue et al. A direct aspiration, first pass technique (ADAPT) versus stent retrievers for acute stroke therapy: An observational comparative study. American Journal of Neuroradiology 2016; 37(10):1860-1865.

[13] Bonnette M, Morris D. Thrombectomy catheter system (patent US2014303658A1).

[14] Cosigny P. Spiral thrombectomy catheter (patent US2013060206A1).

[15] Milner K, Barnett A. Thrombectomy catheter with flow directing mechanism (patent US2015133973A1).

[16] Pearce G, Perkinson ND. Biomechanical probe international patent corporate treatise (WO2006120464); European patent (ep1893195 (a2)); Japanese patent (jp2008639924 (t)); Chinese patent (cn101208049 (a)).

[17] Pearce G, Brookfield P, Burley M, Murcott D, Perkinson ND, Spence J, Wong J. The 'GP' mechanical thrombectomy device: Measurements and modelling, using laboratory models, applications of geometric algebraic techniques and cadaveric brains. Journal of Stroke and Cerebrovascular Disease. 2009; 18(4) 288-293.

[18] Tennuci C, Pearce G, Wong J, Nayak S, Jones T, Roffe C. Comparison of the effectiveness of three methods of recanalization in a model of the middle cerebral artery: Thrombus aspiration via a 4F catheter, thrombus aspiration via the GP thromboaspiration device and mechanical thrombectomy using the solitaire thrombectomy device. Stroke Research and Treatment. 2011;186424.

[19] Goswami P, Mandal DK, Manna NK, Chakrabarti S. Numerical investigations of various aspects of plaque deposition through constricted artery. Journal of Mechanical Engineering and Sciences. 2019; 13(3) 5306:5322.

[20] Chitsaz A, Nejat A, Nouri R. Three-dimensional numerical simulations of aspiration process: Evaluation of two penumbra aspiration catheters performance. Artificial Organs. 2018;42(12):406-419.

[21] Al-Dulaimi MJ, Hamad FA, Rasool AAA, Ameen KA. Effect of sand particles on flow structure of free jet from a nozzle. Journal of Mechanical Engineering and Sciences. 2019;13(3):5542:5561.

[22] Romero G, Higuera I, Félez J, Pearce G, Perkinson ND. Modelling and simulation of a thrombectomy probe applied to the middle cerebral artery by using the bond graph technique. $9^{\text {th }}$ International Conference on Bond Graph Modeling and Simulation. 2010; 172-179.

[23] Romero G, Martinez ML, Pearce G, Mera JM. An investigation into the performance of a new mechanical thrombectomy device using bond graph modeling: application to the extraction of blood clots in the middle cerebral artery. Simulation. 2013; 89(3):381-391. 